\title{
Trigger Algorithms for Alignment and Calibration at the CMS Experiment
}

\author{
Thiago R. F. P. Tomei* \\ On behalf of the CMS Collaboration \\ Universidade Estadual Paulista \\ E-mail: Thiago.Tomei@cern.ch
}

The data needs of the Alignment and Calibration group at the CMS experiment are reasonably different from those of the physics studies groups. Data are taken at CMS through the online event selection system, which is implemented in two steps. The Level-1 Trigger is implemented on custom-made electronics and dedicated to analyse the detector information at a coarse-grained scale, while the High Level Trigger (HLT) is implemented as a series of software algorithms, running in a computing farm, that have access to the full detector information. In this paper we describe the set of trigger algorithms that is deployed to address the needs of the Alignment and Calibration group, how it fits in the general infrastructure of the HLT, and how it feeds the Prompt Calibration Loop (PCL), allowing for a fast turnaround for the alignment and calibration constants.

38th International Conference on High Energy Physics 3-10 August 2016

Chicago, USA

${ }^{*}$ Speaker. 


\section{Alignment and Calibration Triggers}

The good physics performance of CMS depends on the detector being properly aligned and calibrated, such that the data reconstruction may deliver accurate physics objects. To that end, during both cosmics and collisions operation, a certain amount of bandwidth is allocated to the collection of special classes of events for the alignment and calibration algorithms. As every data event recorded by CMS, those are collected by dedicated trigger algorithms (trigger paths) that are part of the HLT menu. Figure 1 shows the bandwidth distribution for the different data streams collected by CMS for a run from the first half of 2016; the accompanying table showcases some of the trigger algorithms used by each subdetector in the experiment.

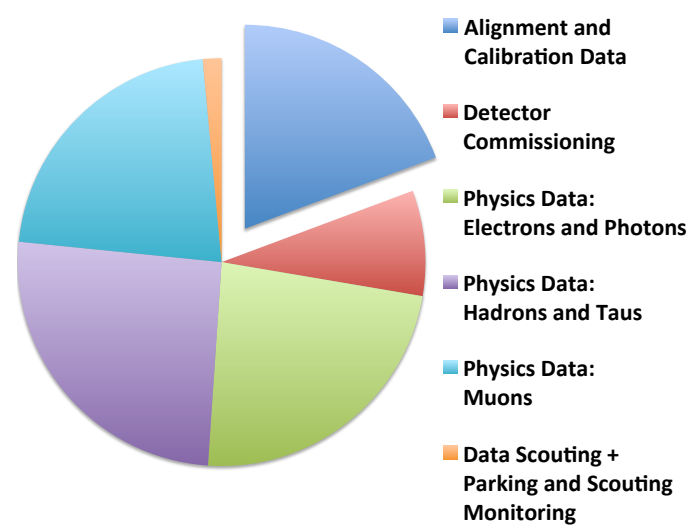

\begin{tabular}{|c|c|}
\hline \multicolumn{2}{|c|}{ HLT algorithms for subdetectors } \\
\hline TRACKER & ECAL \\
\hline Random triggers & $\eta, \pi^{0}$ triggers \\
\hline Zero bias & $\phi$-symmetry \\
\hline Isolated / leading bunch & Single, double $e$ \\
\hline HCAL & MUON \\
\hline Non-zero suppressed & RPC monitoring \\
\hline$\phi$-symmetry & Single, double $\mu$ \\
\hline Isolated tracks & Cosmic ray triggers \\
\hline
\end{tabular}

Figure 1: Collected data are grouped in streams, with dedicated streams for alignment and calibration needs, and streams for physics data, commissioning and monitoring (chart). The needs of the subsystems are addressed through different HLT algorithms (table).

Generally, for alignment and calibration purposes, there exist three classes of trigger algorithms, which are divided in the following streams:

- Express Stream: a low rate stream with trigger paths that feed the Prompt Calibration Loop (PCL) as explained in Sec. 2. In general, this stream is built to accept events at approximately $50 \mathrm{~Hz}$, to be compared with the nominal HLT output of $1 \mathrm{kHz}$.

- AICA Streams: a stream formed by trigger paths that save a reduced event content - only a subset of the raw detector data. Because of the smaller event size, these streams are allowed to accept events at a much higher rate than the nominal HLT output.

- Physics Streams: the HLT algorithms present in the trigger menu for physics measurements are also leveraged for alignment and calibration purposes.

\section{Prompt Calibration Loop}

The PCL, illustrated in Fig. 2, is embedded in the CMS data-taking workflow and is executed for each data-taking run. Starting from the Express Stream, a set of compact datasets named AlCaReco are produced automatically, with reduced event content, for input to the alignment and calibration algorithms. The resulting calibration conditions are transferred to the online environment of the CMS detector site via a database system. The PCL comprises the following steps: 


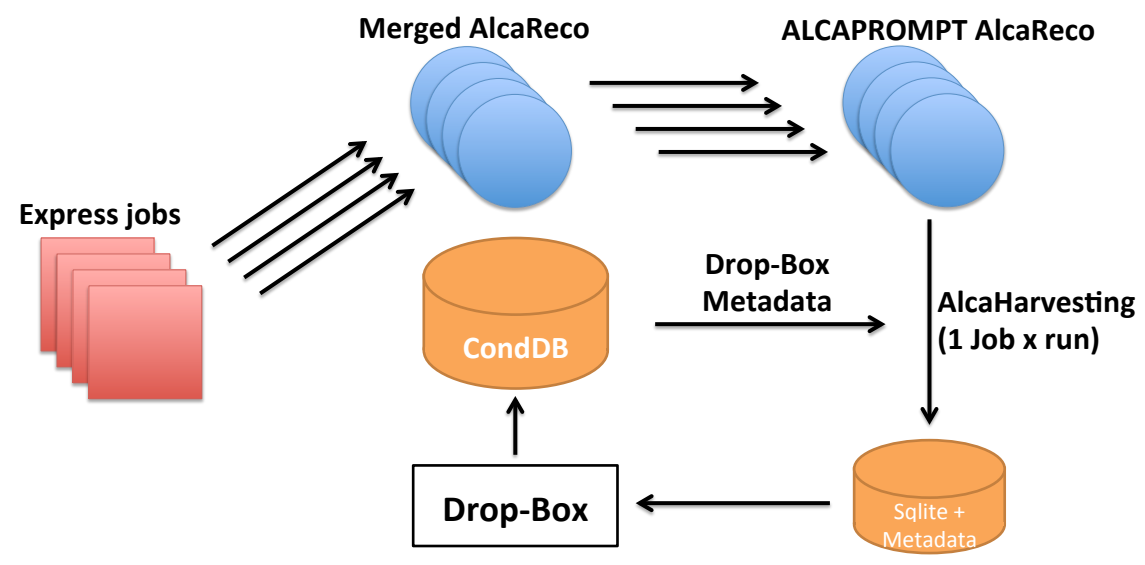

Figure 2: The Prompt Calibration Loop as executed in the CMS data-taking procedure.

- Express Processing: a set of parallel jobs are run over the Express Stream; the need for parallelisation is due to the short latency allowed for this procedure - a maximum of a couple of hours. The outputs of all the jobs are merged into the aforementioned AlCaReco datasets.

- AlCaReco Splitting: a second set of jobs run the dedicated, CPU-intensive computation of the alignment and calibration constants. These jobs also run in parallel, with each job processing a given number of luminosity sections ${ }^{1}$. Their aggregated outputs are the socalled ALCAPROMPT files, which contain the summary information for the quantities of interest in the form of histograms.

- AlCa Harvesting: a single job processes the whole set of ALCAPROMPT files for that run and stores the derived conditions in a database file (SQlite). Aditionally, the CMS software framework automatically produces a job report from which the conditions metadata are derived.

- Conditions Upload: the database files are uploaded to the main conditions database of the experiment automatically. Each PCL workflow produces at least one database file; the different PCL workflows are described in Sec. 3.

\section{Alignment and Calibration in PCL}

The following workflows are part of the Prompt Calibration Loop:

- The 3D position, dimension and orientation of the luminous region at CMS is determined, as function of time, from the set of reconstructed event vertices. This determination is made through a fitting procedure that can be done up to once every luminosity section.

- The Silicon Strip Tracker is monitored both for the detection of problematic channels and for the determination of the gains of each channel. For each of those workflows, a set of calibration conditions is determined per run.

- The Silicon Pixel Tracker is monitored for involuntary movement of the larger structures, and its geometry is aligned based on the reconstructed tracks. A new geometry alignment can be determined for each run, if necessary.

\footnotetext{
${ }^{1} \mathrm{~A}$ luminosity section is the quantum of data-taking in CMS and corresponds to $23.31 \mathrm{~s}$.
} 
Additionally, the transparency loss on the Electromagnetic Calorimeter (ECAL) due to radiation damage is monitored in a quasi-online manner; the calibration algorithms are run on dedicated online resources at the CMS experiment location. One measurement is done approximately every 30 minutes.

Thanks to the PCL, the best calibrations are available in 1-2h for prompt data reconstruction. With the current data volumes, and re-reconstruction campaigns limited to no more than once a year, CMS physics performance depends on it.

\section{Results}

Analyses presented at this ICHEP were able to use the $12.9 \mathrm{fb}^{-1}$ dataset collected until July $15^{\text {th }}$ with the latest calibrations, thanks to the Prompt Calibration Loop. Detector Performance Groups collected a significant number of events for alignment and calibration that will be used to deliver the ultimate conditions for 2016 data, to be used in future re-reconstruction campaigns.


Figure 3: Alignment and calibration results: distance in the transverse plane of the track at its closest approach to a refit unbiased primary vertex (left) and residual miscalibration of the ECAL barrel (EB) channel inter-calibration (right).

\section{Acknowledgements}

This material is based upon work supported in part by the São Paulo Research Foundation (FAPESP) under Grants No. 2013/01907-0, 2013/15479-0, and 2015/004 17-4.

\section{References}

[1] CMS Collaboration, "The CMS experiment at the CERN LHC", JINST 3 (2008) S08004.

[2] G. Cerminara and B. van Besien, "Automated workflows for critical time-dependent calibrations at the CMS experiment”, J. Phys. Conf. Ser. 664, no. 7, 072009 (2015). 